\title{
ARTICLE The role of miR-155 in cigarette smoke-induced pulmonary inflammation and COPD
}

\author{
E. G. De Smet ${ }^{1}$, H. P. Van Eeckhoutte ${ }^{1}$, F. Avila Cobos ${ }^{2}$, E. Blomme ${ }^{1}$, F. M. Verhamme ${ }^{1}$, S. Provoost ${ }^{1}$, S. E. Verleden ${ }^{3}$, K. Venken ${ }^{4,5}$, T. Maes ${ }^{1}$,
} G. F. Joos ${ }^{1}$, P. Mestdagh ${ }^{2}$, G. G. Brusselle ${ }^{1}$ and K. R. Bracke ${ }^{1}$

Chronic obstructive pulmonary disease (COPD) is a highly prevalent respiratory disease characterized by airflow limitation and chronic inflammation. MiR-155 is described as an ancient regulator of the immune system. Our objective was to establish a role for miR-155 in cigarette smoke (CS)-induced inflammation and COPD. We demonstrate increased miR-155 expression by RT-qPCR in lung tissue of smokers without airflow limitation and patients with COPD compared to never smokers and in lung tissue and alveolar macrophages of CS-exposed mice compared to air-exposed mice. In addition, we exposed wild type and miR-155 deficient mice to CS and show an attenuated inflammatory profile in the latter. Alveolar macrophages were sorted by FACS from the different experimental groups and their gene expression profile was analyzed by RNA sequencing. This analysis revealed increased expression of miR-155 targets and an attenuation of the CS-induced increase in inflammation-related genes in miR-155 deficient mice. Moreover, intranasal instillation of a specific miR-155 inhibitor attenuated the CS-induced pulmonary inflammation in mice. Finally, elastase-induced emphysema and lung functional changes were significantly attenuated in miR-155 deficient mice. In conclusion, we highlight a role for miR-155 in CS-induced inflammation and the pathogenesis of COPD, implicating miR-155 as a new therapeutic target in COPD.

Mucosal Immunology (2020) 13:423-436; https://doi.org/10.1038/s41385-019-0241-6

\section{INTRODUCTION}

Chronic obstructive pulmonary disease (COPD) is a highly prevalent respiratory disease with cigarette smoking as the main risk factor. ${ }^{1}$ It is the fourth leading cause of death in the world ${ }^{2}$ and it is estimated that its morbidity and mortality rate will increase even further in the following years. ${ }^{3}$ Many mechanistic concepts leading to the development of COPD have been proposed. A prominent pathological feature of COPD is the presence of chronic exaggerated inflammation in the lungs after exposure to cigarette smoke (CS). ${ }^{4}$ Chemokine and cytokine signaling pathways are essential for the influx of immune cells involved in this process. In addition, the accumulation of these inflammatory cells contributes to pulmonary damage as endogenous anti-proteinases cannot neutralize the high concentrations of proteases, such as neutrophil elastase and MMP12, that are released. ${ }^{5}$ On top of this, oxidative stress caused by CS activates transcription factors such as nuclear factor $\kappa B$ (NFkB), which enhances the inflammatory response by positively regulating the expression of pro-inflammatory genes. ${ }^{6}$

MicroRNAs (miRNAs) are short, non-coding single stranded RNA molecules that are involved in the regulation of gene expression. They are known to play a role in many fundamental biological processes, including immunity and inflammation. MicroRNA-155 (miR-155) is described as an ancient regulator of the immune system. ${ }^{7}$ MiR-155 is expressed in cells of both the innate and adaptive immune system, where its expression is low under normal homeostatic conditions and becomes induced upon stimulation. $^{8-11}$ Studies with miR-155 deficient animals have established a role for miR-155 in the inflammatory response, given that miR-155 deficiency confers protection against various experimental (auto-)immune disorders. ${ }^{12-16}$

MiR-155 deficiency has already been described as beneficial in models of allergic airway inflammation, where miR-155 is necessary for interleukin (IL)-33 induced innate lymphoid cell (ILC) type 2 expansion, eosinophilic airway inflammation and allergen-induced maturation and T cell priming of dendritic cells (DCs). ${ }^{17-19}$ Next to its role in T cells, miR-155 has also been linked to macrophage function. ${ }^{20-22}$ Macrophages play a major role in orchestrating the inflammatory response in $\mathrm{COPD}^{23}$ activated macrophages from COPD patients excessively release inflammatory mediators such as $\mathrm{IL}-1 \beta, \mathrm{IL}-6$, tumor necrosis factor (TNF), CXCL1 and CCL2, ${ }^{24}$ as well as matrix metalloproteases (such as MMP-9 and MMP-12). ${ }^{25,26}$

In miRNA profiling studies, we have previously reported increased expression of miR-155 in lungs of smokers without airflow limitation and patients with mild to moderate COPD $^{27}$ as well as in lungs of CS-exposed mice. ${ }^{28}$ In the present study, we have validated these results in a larger cohort, including also patients with severe to very severe COPD. Since the functional role of miR-155 in the pathogenesis of COPD has not yet been

\footnotetext{
'Laboratory for Translational Research in Obstructive Pulmonary Diseases, Department of Respiratory Medicine, Ghent University Hospital, Ghent, Belgium; ${ }^{2}$ Center for Medical Genetics, Ghent University, Ghent, Belgium; ${ }^{3}$ Department of Respiratory Medicine, University Hospital Gasthuisberg, Catholic University of Leuven, Leuven, Belgium; ${ }^{4}$ Laboratory

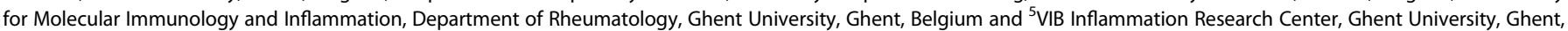
Belgium

Correspondence: K. R. Bracke (ken.bracke@UGent.be)
}

Received: 18 December 2018 Revised: 22 November 2019 Accepted: 23 November 2019

Published online: 9 December 2019 
424

investigated, we exposed wild type and miR-155 KO mice to CS and quantified different inflammatory cell types, chemokines and cytokines in the bronchoalveolar lavage (BAL) fluid and lungs. In addition, to gain insight in the genes and pathways affected by miR-155 deficiency, we sorted alveolar macrophages by FACS from the different experimental groups and analyzed their gene expression profile by RNA sequencing. Furthermore, we treated mice with a specific miR-155 inhibitor and investigated the effect on CS-induced inflammation. Finally, to elucidate the role of miR-155 in development of pulmonary emphysema and lung functional changes, we investigated wild type and miR-155 deficient mice in an elastase model of emphysema.

\section{RESULTS}

MiR-155-5p expression in lungs of never smokers, smokers without airflow limitation, and patients with COPD

We evaluated the expression of hsa-miR-155-5p (further referred to as miR-155) in lung tissue of 92 human subjects, including 18 never smokers, 26 smokers without airflow limitation, 34 patients with COPD GOLD II, and 14 patients with COPD GOLD III-IV. Subject characteristics are reported in Table 1. MiR-155 expression was significantly increased in lung tissue of smokers and patients with COPD compared to never smokers. In addition, the expression in COPD GOLD III-IV patients was significantly higher compared to smokers and COPD GOLD II patients (Fig. 1a). Furthermore, in COPD patients miR-155 expression was significantly and inversely correlated with the severity of airflow limitation (forced expiratory volume in $1 \mathrm{~s}$ (FEV1) \% predicted and FEV1/ forced vital capacity (FVC) post-bronchodilator) and with diffusing capacity of the lung for carbon monoxide ( $D L_{c o}$ ) (Fig. $1 b-d$ ). These data thus demonstrate that pulmonary expression of miR-155 is increased in smokers and is further amplified in patients with severe COPD.

\section{MiR-155-5p expression is increased in lung tissue and alveolar} macrophages of mice exposed to CS

To measure the effect of CS exposure on the expression of mmumiR-155-5p (further referred to as miR-155), we performed RTqPCR on lung tissue of WT mice exposed to air or CS for four or 24 weeks and on alveolar macrophages sorted from WT mice exposed to air or CS for 4 weeks. MiR-155 expression was significantly increased in lung tissue and in FACS sorted alveolar macrophages of mice exposed to CS compared to air-exposed mice (Fig. $1 \mathrm{f}-\mathrm{g}$ ). Importantly, after 4 weeks of CS-exposure the increase in miR-155 was more prominent in alveolar macrophages than in lung tissue, with a fold change of 4.6 versus 1.6, respectively.

MiR-155 deficiency attenuates CS-induced inflammation To evaluate the role of miR-155 in CS-induced inflammation, we exposed WT and miR-155 knock-out (KO) mice to 4 weeks of air or CS. In BAL of miR-155 KO mice, the CS-induced increase in numbers of neutrophils, CD $11 \mathrm{~b}^{+}$and $\mathrm{CD} 103^{+} \mathrm{DCs}$ and $\mathrm{CD} 8^{+}$ and $\mathrm{CD}^{+}{ }^{+} \mathrm{T}$ lymphocytes was significantly attenuated compared to WT mice (Fig. $2 c-g$ ). Intracellular staining for IFN- $\gamma$, IL13 and IL-17 within the $\mathrm{CD}^{+}{ }^{+} \mathrm{T}$ lymphocyte population demonstrated an increase after CS exposure of all $T$ helper (Th1, Th2, and Th17) subsets in WT mice, with the highest increase in the Th1 and Th17 subpopulations. Mice deficient for miR-155 showed a significantly attenuated increase of these three $T$ helper subsets upon CS exposure compared with WT mice (Fig. $2 \mathrm{~h}-\mathrm{j}$ ). Likewise, in lung tissue of miR-155 KO mice, the CS-induced increase in percentages of $\mathrm{CD}_{1} 1 \mathrm{~b}^{+}$and $\mathrm{CD}_{103}{ }^{+} \mathrm{DCs}$ and $\mathrm{CD}_{4}^{+}, \mathrm{CD} 9^{+} \mathrm{CD}_{4}{ }^{+}$, and $\mathrm{CD} 9^{+} \mathrm{CD} 8^{+} \mathrm{T}$ lymphocytes was significantly attenuated compared to WT mice (See Supplementary Fig. S1).
MiR-155 deficiency attenuates cytokine and chemokine levels in BAL and lung tissue after 4 weeks of CS exposure

Given the attenuated increase in inflammatory cell types in the BAL fluid and lung tissue of miR-155 KO animals, we measured the expression of various chemokines and cytokines involved in the differentiation and recruitment of these inflammatory immune cells (Supplementary Tables S1-S3). The CS-induced increase in CXCL1, CCL2, TNF, IL-6, IL-1 $\beta$, IFN- $\gamma$, and IL-17A protein expression in the BAL fluid of WT mice was significantly attenuated in the miR-155 KO mice (Fig. 3). In addition, also in lung tissue, the increase in IL-6 and TNF protein expression and CXCL1, CXCL2, CCL2, and IL- 6 mRNA expression in CS-exposed WT mice was significantly attenuated in miR-155 KO mice (Supplementary Tables S2-S3).

Gene expression profiling of sorted alveolar macrophages from air and CS-exposed WT and miR-155 KO mice

To gain insight in the genes and pathways affected by miR-155 $\mathrm{KO}$, alveolar macrophages from air and CS-exposed WT and miR$155 \mathrm{KO}$ mice were FACS sorted (gated as $\mathrm{CD}_{4} 5^{+}$SiglecF $^{+} \mathrm{CD} 11 \mathrm{c}^{+}$ cells) and poly- $\mathrm{A}^{+}$RNA sequencing was performed on 5 mice per group (NCBI GEO ID: GSE137653). A heat map showing the differential gene expression in the four groups is depicted in Fig. 4a. This heat map demonstrates that the CS-induced differential expression of many genes in WT mice is less pronounced in miR-155 KO mice, recapitulating the attenuation of the inflammatory profile in BAL and lung tissue. Additionally, mice from the four groups (air and CS-exposed WT and miR-155 $\mathrm{KO}$ mice) were grouped based on similarities in the alveolar macrophages' expression profile, revealing an accurate separation between the gene expression profile of macrophages from smoke-exposed WT and miR-155 KO mice (Supplementary Fig. S2).

CS-exposure results in differential expression of 656 genes in WT macrophages (upregulation of 421 and downregulation of 235 genes) and 415 genes in miR-155 KO macrophages (upregulation of 275 genes and downregulation of 140 genes) $(\operatorname{abs}(\log 2 \mathrm{FC}) \geq 1$ and $p$-value $<0.05$, Supplementary Fig. S3).

When comparing the expression profile of macrophages from air-exposed WT and miR-155 KO mice, eight genes were significantly lower expressed and ten genes were significantly higher expressed in the miR-155 KO macrophages, including four predicted targets of mmu-miR-155: cyclin-dependent kinase-like 2 (cdkl2); ATPase, class V, type 10D (atp10d); endothelin converting enzyme 1 (ece1) and Ras association (RalGDS/AF-6) domain family member 6 (rassf6) ${ }^{29}$ (abs(log2FC) $\geq 1$ and $p$-value $<0.05$, Fig. $\left.4 b\right)$.

After CS-exposure 38 genes were lower expressed and 15 genes were higher expressed in the miR-155 KO macrophages compared to the WT macrophages (Table 2, Fig. 4c). Five of the significantly higher expressed genes were predicted targets of mmu-miR-155: cdkl2; atp10d; rassf6; $\mathrm{Y}$-aminobutyric acid B receptor 1 (gabbr1) and solute carrier family 10 member 6 (slc10a6). ${ }^{29}$ Rassf6, a predicted target of miR-155 and a known NF-kB inhibitor ${ }^{30}$ showed a non-significant downregulation after CS-exposure in WT macrophages, but showed up to 40-fold higher levels in miR-155 KO macrophages (Fig. 5a). However, a luciferase reporter assay could not confirm rassf6 as a direct target of miR-155 (data not shown). Interestingly, the CS-induced upregulation of several inflammatory genes and other genes previously linked to COPD pathogenesis, including chemokine-like receptor 1 (Cmklr1); C1qa (complement component 1, q subcomponent, alpha polypeptide); a disintegrin and metallopeptidase domain 19 (adam19) and matrix metallopeptidase 12 ( $\mathrm{mmp} 12$ ) was significantly attenuated in miR-155 KO macrophages, compared to WT macrophages (Fig. 5b-e).

Gene set enrichment analysis

We performed gene set enrichment analysis (GSEA) analysis using the 13225 genes that were expressed in sorted alveolar 
The role of miR-155 in cigarette smoke-induced pulmonary inflammation and...

Table 1. Characteristics of study population (RT-qPCR study) $(n=92)$.

\begin{tabular}{|c|c|c|c|c|}
\hline & Never smokers & Smokers without COPD & COPD GOLD ॥ & COPD GOLD III-IV \\
\hline Gender ratio (male/female) & $6 / 12 \#$ & 19/7\# & $31 / 3 \#$ & $8 / 6 \#$ \\
\hline Current-/ex-smoker & - & $16 / 10$ & $22 / 12$ & $0 / 14$ \\
\hline Smoking history (PY) & $0(0-0)$ & $28(15-45)^{*}$ & $45(40-60)^{*} \S$ & $30(25-30)^{*}+$ \\
\hline $\mathrm{FEV}_{1}$ post $(\mathrm{L})$ & $2,7(2.3-3.2)$ & $2,7(2.3-3.3)$ & $2.0(1.8-2.4)^{*} \S$ & $0.7(0.7-0.9)^{*} \S \dagger$ \\
\hline $\mathrm{FEV}_{1} / \mathrm{FVC}$ post & $78(75-83)$ & $75(71-79)^{*}$ & $56(53-60)^{*} \S$ & $32(27-35)^{*} \S \dagger$ \\
\hline $\mathrm{DL}_{\mathrm{CO}}$ (\% predicted) & $90(80-105)$ & $80(61-102)$ & $67(51-87)^{*}$ & $35(33-41)^{*} \S \dagger$ \\
\hline $\mathrm{K}_{\mathrm{CO}}(\%$ predicted $)$ & $103(88-123)$ & $91(68-107)^{*}$ & $87(62-108)^{*}$ & $59(50-65)^{*} \S \dagger$ \\
\hline ICS (yes/no) & $0 / 18 \#$ & $1 / 25 \#$ & 15/19\# & 13/1\# \\
\hline
\end{tabular}

$P Y$ pack years, $F E V_{1}$ forced expiratory volume in $1 \mathrm{~s}, F V C$ forced vital capacity, $D L_{C O}$ diffusing capacity of the lung for carbon monoxide, ICS inhaled corticosteroids

Data are presented as median (IQR)

Mann-Whitney $U$ test: ${ }^{*} P<0.05$ versus never smokers; $\S P<0.05$ versus smokers without COPD; $+P<0.05$ versus COPD GOLD II

Fisher's exact test: $\# P<0.001$

macrophages to discover pathways and functions associated with smoke exposure and/or with miR-155 expression. Smoke-exposure resulted in enrichment of genes involved in metabolic pathways (oxidative phosphorylation, metabolism of xenobiotics, amino sugar and nucleotide sugar metabolism, galactose metabolism, proteasome, lysosome, steroid biosynthesis, etc.) and in proliferation (DNA replication, P53 signaling pathway) in both WT and miR155 KO macrophages. However, many of these pathways had a lower enrichment score in CS-exposed miR-155 KO macrophages compared to CS-exposed WT macrophages. This was also true for genes involved in pro-inflammatory immune signaling pathways, such as cytokine-cytokine receptor interaction and the chemokine signaling pathway (Supplementary Tables S4-S6).

Inhibition of mmu-miR-155-5p in the lungs of air and CS-exposed mice

By intranasal instillation of an LNA modified mmu-miR-155-5p inhibitor or a scrambled control we investigated the effect of miR155 inhibition on CS-induced inflammation. Upon inhibition of miR-155 the numbers of DCs and $\mathrm{CD}^{+}$and $\mathrm{CD} 8^{+} \mathrm{T}$ cells in the BAL fluid of CS-exposed mice were significantly lower compared to the scrambled control (Fig. $6 \mathrm{~d}-\mathrm{f}$ ). In addition, CCL2 protein levels in the BAL fluid, as measured by ELISA, were significantly lower in mice treated with a miR-155 inhibitor compared to mice treated with scrambled control (Fig. 6g). Importantly, miR-155 expression was almost completely undetectable in lungs of mice treated with the miR-155 inhibitor, demonstrating a successful inhibition (Fig. 6h).

Elastase-induced pulmonary emphysema is attenuated in miR-155 deficient mice

To investigate the effect of miR-155 deficiency on development of pulmonary emphysema and lung functional changes we intratracheally administered porcine pancreatic elastase (PPE) to mice. In a first protocol we quantified emphysema and inflammation in BAL fluid 7 days after a single intratracheal instillation of elastase. Elastase-induced development of emphysema, as measured by an increased mean linear intercept $(\mathrm{Lm})$, was significantly attenuated in miR-155 KO mice (Fig. 7a-e). Also, the increase in BAL neutrophils, DCs, and lymphocytes upon instillation of elastase was attenuated in miR-155 KO mice (Fig. 7f-h).

In a second protocol mice received an instillation of elastase for three consecutive weeks and were subjected to in vivo lung function measurements 7 days after the third and last instillation of elastase. Importantly, the elastase-induced increase in compliance and reduction in elasticity, were significantly attenuated in miR-155 KO mice (Fig. 7i, j). Moreover, the left-upward shift in the pressure volume-loops of elastase-treated mice (which is reminiscent of emphysema), was also significantly attenuated in miR-155 $\mathrm{KO}$ mice (Fig. 7k).

\section{DISCUSSION}

In this translational study we first demonstrated increased expression of miR-155 in lungs of smokers and patients with (moderate and especially severe) COPD, as well as in lungs and alveolar macrophages of CS-exposed mice. Next, we showed that CS-induced pulmonary inflammation is significantly attenuated in miR-155 deficient mice, since key inflammatory cells, cytokines, and chemokines are reduced in BAL fluid of CS-exposed miR-155 KO mice. RNA sequencing in alveolar macrophages revealed differential expression of miR-155 targets in alveolar macrophages according to genotype (WT versus miR-155 KO) and/or exposure (air versus CS). Intriguingly, we discovered an attenuation of the CS-induced increase in key genes involved in the pathogenesis of COPD (encompassing proteases MMP12 and ADAM19) in alveolar macrophages of miR-155 deficient mice. In addition, intranasal instillation of a specific miR-155 inhibitor attenuated the CSinduced pulmonary inflammation in mice. Finally, miR-155 deficiency also protected against elastase-induced emphysema and lung functional changes.

High levels of miR-155 have been reported in various immune diseases and related animal models. ${ }^{14,17,31,32}$ Pathogen and damage-associated molecular patterns (PAMPs/DAMPs), ${ }^{10}$ alarmins $^{33}$ inflammatory stimuli ${ }^{10}$ and hypoxia, ${ }^{34}$ which are all present after smoke exposure and in COPD, are reported to be responsible for induction of miR-155 expression. In large miRNA profiling studies, we previously demonstrated increased miR-155 expression in smokers without airflow limitation and in patients with mild to moderate $\mathrm{COPD}^{27}$ as well as in mice exposed to $\mathrm{CS}^{28}$ In the present study we confirm and expand these results by demonstrating a significantly higher miR-155 expression in patients with severe to very severe COPD. Furthermore, we show that miR-155 expression levels in COPD patients are significantly and inversely correlated with parameters of airflow limitation $\left(\mathrm{FEV}_{1}, \mathrm{FEV}_{1} / \mathrm{FVC}\right)$ and diffusion capacity of the lungs $\left(\mathrm{DL}_{\mathrm{CO}}\right)$. In addition, we report that the CS-induced increase in miR-155 expression is more prominent in mouse alveolar macrophages, 

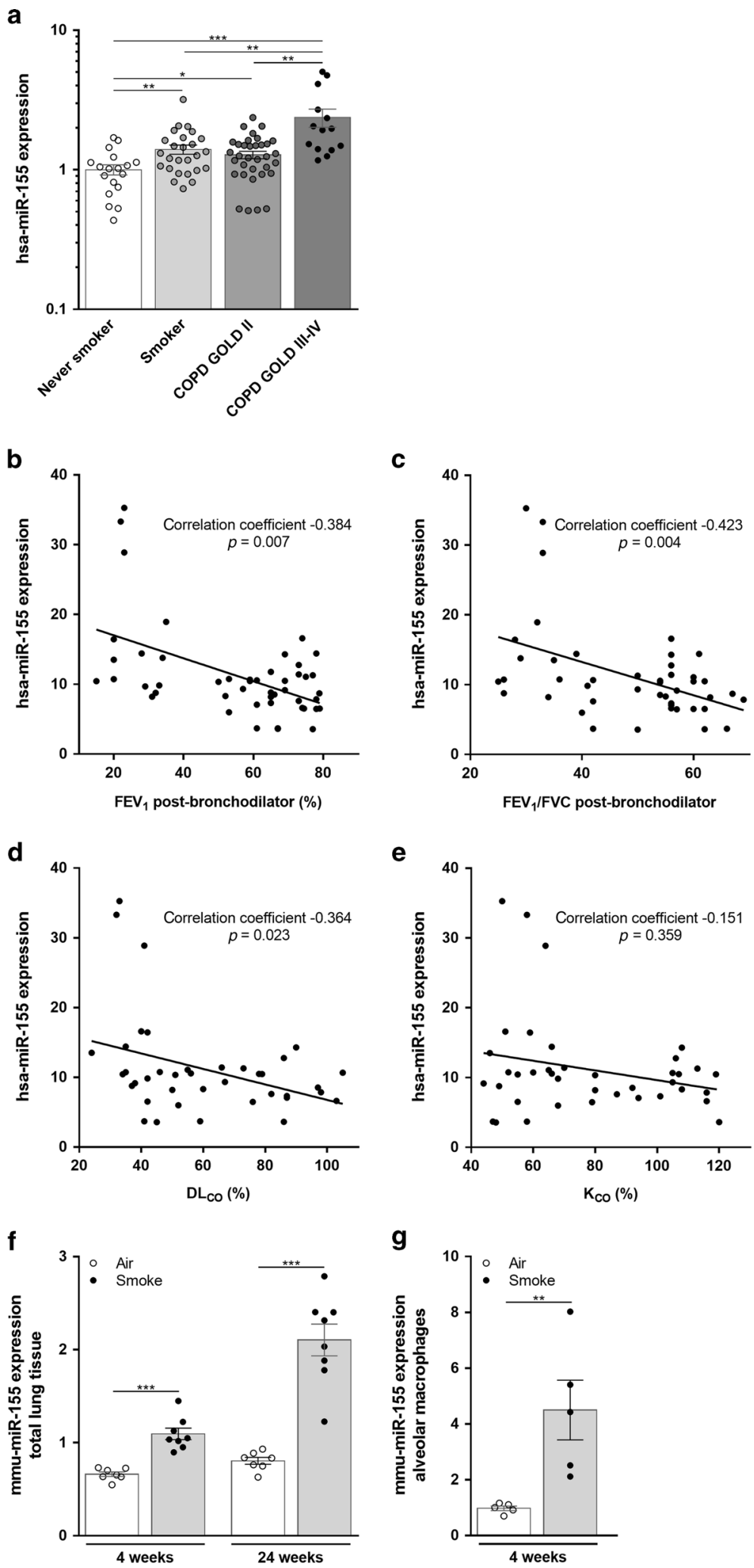

highlighting the alveolar macrophage as an important source of miR-155.

To investigate the functional role of miR-155 in CS-induced inflammation, we exposed WT and miR-155 KO mice to 4 weeks of CS. Data on inflammatory cell numbers and mediators in the BAL fluid and lungs reveal an attenuated inflammatory profile in CSexposed mice when miR-155 expression is absent.

g

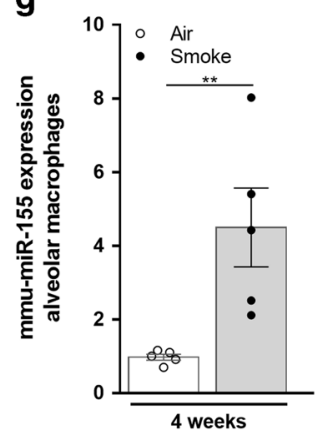

Interestingly, miR-155 promotes chemokine expression and proinflammatory chemokine receptor expression in arthritis monocytes. $^{31}$ This suggests that high levels of miR-155 in proinflammatory immune cells are able to induce chemokine production. Activated macrophages from COPD patients release more of these inflammatory mediators such as IL-1 $\beta, \mathrm{IL}-6, \mathrm{TNF}$, CXCL1, and CCL2. ${ }^{24}$ Also in the murine model of COPD these 
Fig. 1 Hsa-miR-155-5p expression in never smokers, smokers without airflow limitation and patients with COPD and correlation with lung function parameters. Mmu-miR-155 expression in total lung tissue and sorted alveolar macrophages of air and cigarette smokeexposed WT mice. Hsa-miR-155 levels measured by RT-qPCR in lung tissue of never smokers $(n=18)$, smokers without airflow limitation ( $n=$ 26), patients with COPD GOLD II $(n=34)$ and patients with COPD GOLD III-IV $(n=14)$. Data were analyzed using the standard curve method and expression of hsa-miR-155-5p was calculated relative to the expression of a small nucleolar RNA (RNU48) (a). Spearman correlation analysis in COPD patients of hsa-miR-155-5p levels with post-bronchodilator values of forced expiratory volume in $1 \mathrm{~s}\left(\mathrm{FEV} \mathrm{V}_{1}\right)(\mathbf{b})$, and the ratio of $\mathrm{FEV}_{1}$ to forced vital capacity $\left(\mathrm{FEV}_{1} / \mathrm{FVC}\right)(\mathbf{c})$, carbon monoxide diffusing capacity $\left(\mathrm{DL}_{\mathrm{CO}}\right)(\mathbf{d})$ and the ratio of $\mathrm{DL}_{\mathrm{CO}}$ to alveolar volume $\left(\mathrm{K}_{\mathrm{CO}}\right)(\mathbf{e})$. Mmu-miR-155-5p levels measured by RT-qPCR in lung tissue of WT mice exposed to air or cigarette smoke for four or 24 weeks. Data were analyzed using the standard curve method and expression of mmu-miR-155-5p was calculated relative to the expression of two endogenous control genes (SNORD68 and SNORD95) ( $n=7-8$ animals/group) (f). Mmu-miR-155-5p levels measured by RT-qPCR in sorted alveolar macrophages (gated as $\mathrm{CD}_{4} 5^{+}$SiglecF ${ }^{+} \mathrm{CD} 11 \mathrm{c}^{+}$) of WT mice exposed to air or cigarette smoke for 4 weeks. The expression of two endogenous control genes (snoRNA-55 and U6 snRNA) was measured and the $\Delta \Delta$ Ct-method was used to calculate the expression of mmu-miR-155-5p $\left(n=7-8\right.$ animals/group) $(\mathbf{g})$. Data are expressed as mean \pm SEM $\left({ }^{* *} P<0.01,{ }^{* * *} P<0.001\right)$.
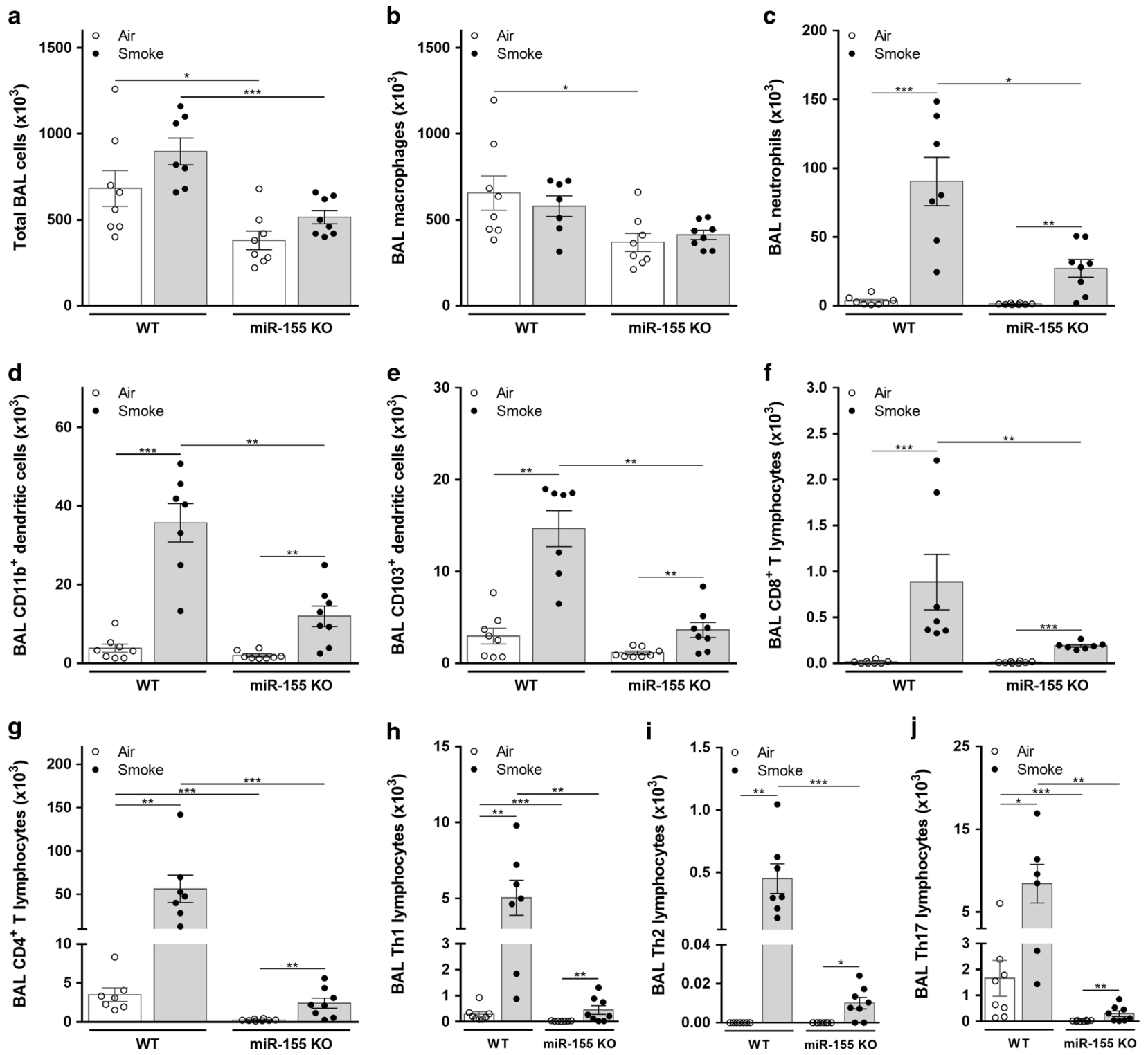

Fig. 2 Inflammatory cell numbers in the bronchoalveolar lavage fluid of WT and miR-155 KO mice exposed to air or cigarette smoke for four weeks. Total BAL cells (a) and measurement by flow cytometry of alveolar macrophages (b), neutrophils (c), CD11 b ${ }^{+}$dendritic cells (d), $\mathrm{CD}_{103^{+}}$dendritic cells (e), CD8 ${ }^{+} \mathrm{T}$ lymphocytes (f), CD4 ${ }^{+} \mathrm{T}$ lymphocytes (g) and T helper subsets, Th1 (h), Th2 (i) and Th17 (j) in the bronchoalveolar lavage fluid of WT and miR-155 KO mice exposed to air or cigarette smoke for 4 weeks ( $n=7-8$ animals/group). Gating

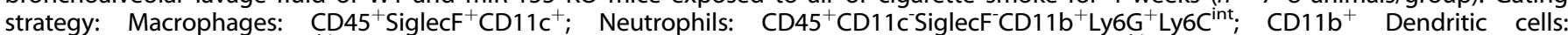

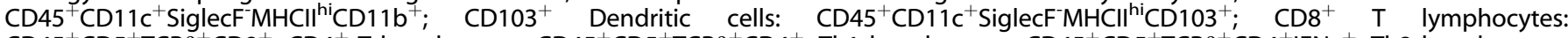
$\mathrm{CD}^{+} 5^{+} \mathrm{CD}^{+} \mathrm{TCR}^{+} \mathrm{CD}^{+}{ }^{+} \mathrm{CD}^{+} \mathrm{T}$ lymphocytes: $\mathrm{CD} 45^{+} \mathrm{CD} 5^{+} \mathrm{TCR} \beta^{+} \mathrm{CD} 4^{+}$; Th1 lymphocytes: $\mathrm{CD} 45^{+} \mathrm{CD} 5^{+} \mathrm{TCR} \beta^{+} \mathrm{CD} 4^{+}$IFN $\gamma^{+}$; Th2 lymphocytes: $\mathrm{CD} 5^{+} \mathrm{CD}^{+} \mathrm{TCR}^{+} \mathrm{CD}^{+}{ }^{+} \mathrm{IL} 13^{+}$; Th17 lymphocytes: $\mathrm{CD} 45^{+} \mathrm{CD} 5^{+} \mathrm{TCR} \beta^{+} \mathrm{CD} 4^{+} \mathrm{IL} 17$. Data are analyzed with the Kruskal-Wallis and Mann-Whitney $U$ test and expressed as mean \pm SEM $\left({ }^{*} P<0.05,{ }^{* *} P<0.01,{ }^{* * *} P<0.001\right)$. 

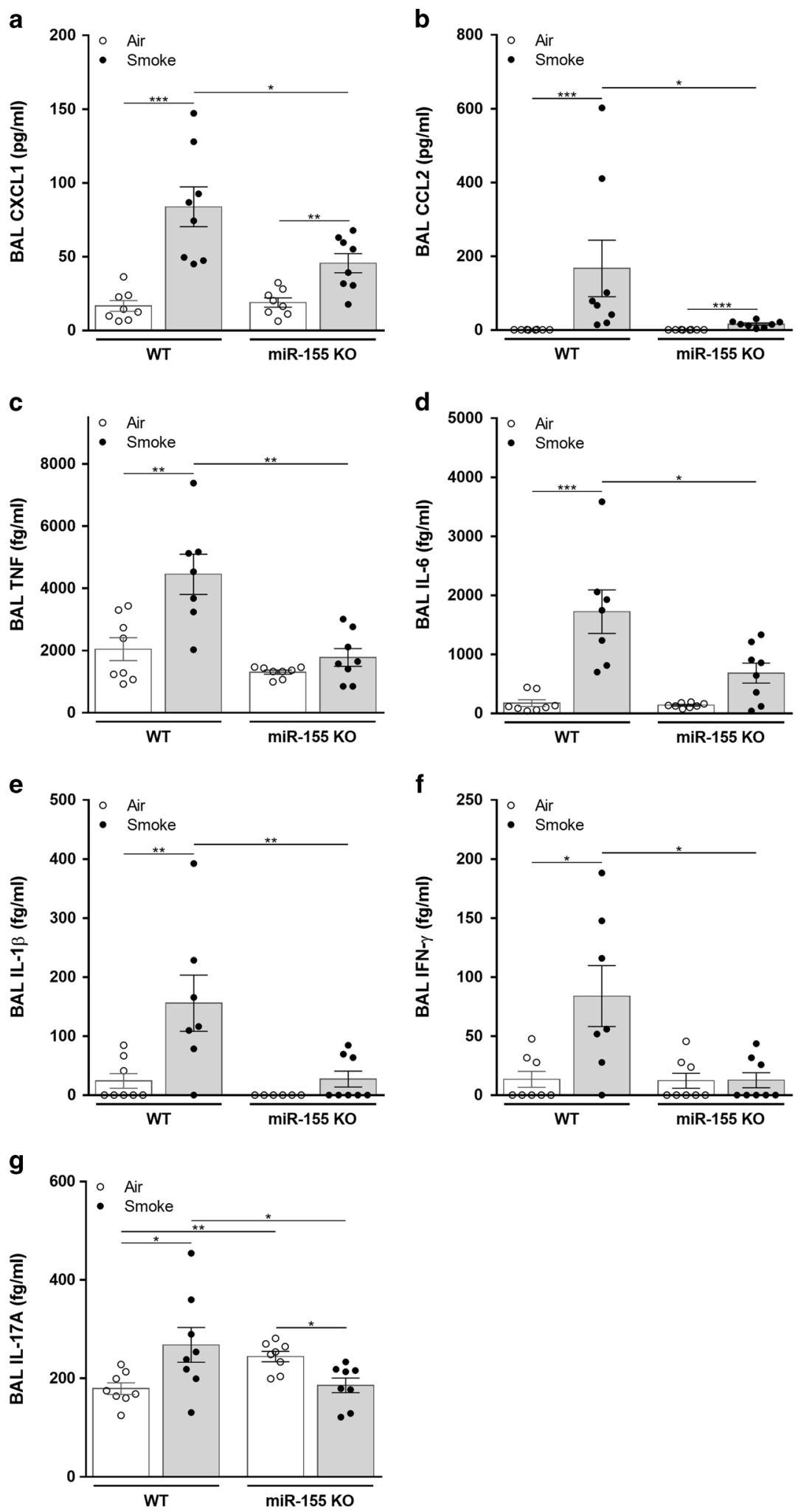

Fig. 3 Effect of miR-155 deficiency on cytokine/chemokine protein levels in BAL supernatants. CXCL1 (a) and CCL2 (b) protein levels in BAL supernatants were measured by ELISA in WT and miR-155 KO mice upon 4 weeks of air or cigarette smoke exposure. TNF (c), IL-6 (d), IL-1 $\beta$ (e), IFN- $\gamma(\mathbf{f})$, and IL-17A (g) protein levels in BAL supernatants were measured by cytometric bead array in WT and miR-155 KO mice upon 4 weeks of air or cigarette smoke exposure $(n=6-8$ animals/group). Data are analyzed with the Kruskal-Wallis and Mann-Whitney $U$ test and expressed as mean \pm SEM $\left({ }^{*} P<0.05,{ }^{* *} P<0.01,{ }^{* * *} P<0.001\right)$. 
a

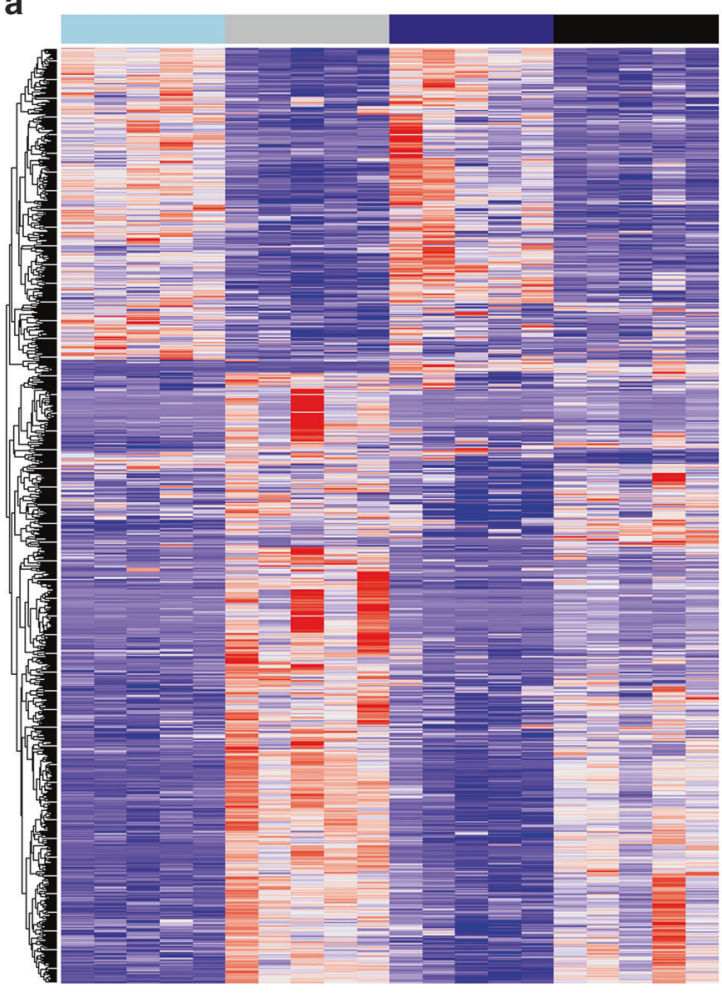

b

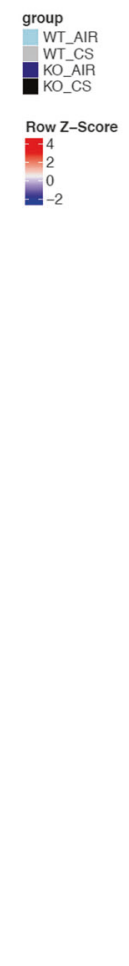

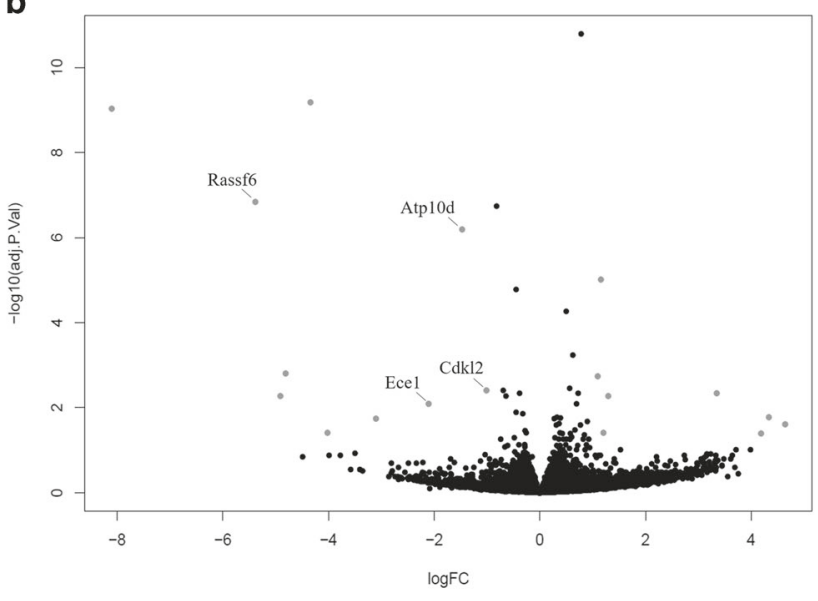

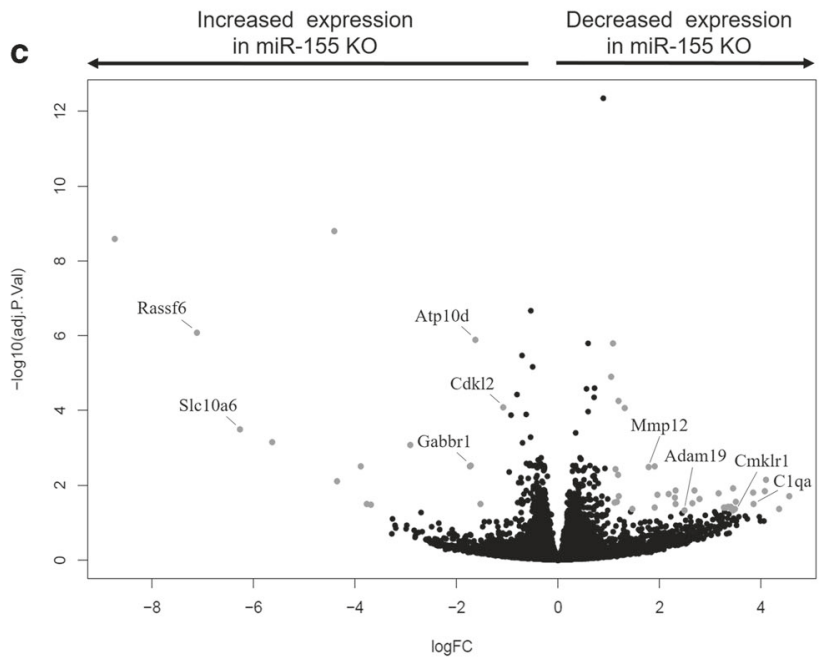

Fig. 4 Poly- $\mathrm{A}^{+}$sequencing of sorted alveolar macrophages from WT and miR-155 deficient mice. Alveolar macrophages were sorted as $\mathrm{CD} 45^{+}$SiglecF ${ }^{+} \mathrm{CD}_{11 \mathrm{c}^{+}}$from bronchoalveolar lavage of WT and miR-155 KO mice exposed to 4 weeks of air or cigarette smoke $(n=5 \mathrm{mice} /$ group). Gene expression analysis of sorted alveolar macrophages was performed by use of poly- $\mathrm{A}^{+}$sequencing. Genes showing log 2 foldchange differences $>1$ (in absolute value) between the different pairwise comparisons and with Benjamini-Hochberg adjusted $p$-values smaller than 0.05 were considered differentially expressed. Heatmap showing differentially expressed genes in any of the four pairwise comparisons (a). Volcano plots of the comparison air-exposed (b) and CS-exposed (c) WT and miR-155 KO mice. Log10 adjusted p-values are plotted against log2 fold change and differentially expressed genes are marked in blue.

mediators are significantly increased in the BAL fluid of CSexposed compared to air-exposed mice. In the BAL fluid of CSexposed miR-155 KO mice, the increase in both the chemotactic mediators and the inflammatory cell numbers is attenuated compared to WT mice, confirming miR-155's role as a proinflammatory non-coding RNA.

MiR-155 expression is increased in macrophages upon inflammation and is known to play an important function in these immune cells. ${ }^{10,20-22,31,35-40}$ Given the high expression of miR-155 in alveolar macrophages in our study and the crucial function of this cell type in COPD pathogenesis, we performed RNA sequencing of FACS sorted alveolar macrophages from WT and miR-155 KO mice exposed to air or CS and analyzed their expression profile in order to gain insights in the pathways affected by miR-155. GSEA of the sorted alveolar macrophages indicates that immune signaling pathways are downregulated in miR-155 KO macrophages. Likewise, differential expression analysis demonstrates increased expression of various inflammatory genes in WT compared to miR-155 KO mice.

C1qa and cmlkr1 are very highly increased in WT macrophages upon $\mathrm{CS}$, while there is almost no increase in miR-155 KO macrophages. C1qa is, next to its function as a recognition component and initiator of the inflammatory complement cascade, also involved in apoptotic cell clearance. ${ }^{41}$ Cmklr1 functions as a receptor for the chemoattractant chemerin and our research group previously demonstrated that cmklr1 $\mathrm{KO}$ mice show an attenuated inflammatory response upon CS exposure. ${ }^{42}$ Also, the induction of mmp12 is significantly attenuated in miR$155 \mathrm{KO}$ macrophages. Mmp12 is critically involved in the development of emphysema, as witnessed by the fact that mmp12-deficient mice are not susceptible to emphysema despite long-term exposure to $\mathrm{CS}^{43}$ In addition, $\mathrm{mmp} 12$ is important for the release of TNF from the cell membrane, generating a biologically active mature protein. ${ }^{44}$ Another interesting gene differentially expressed between CS-exposed WT and miR-155 KO mice is ADAM19. Polymorphisms in this gene are associated with airflow limitation $\left(\mathrm{FEV}_{1} / \mathrm{FVC}\right)$, suggesting a role for $A D A M 19$ in pulmonary function. ${ }^{45}$

When investigating the predicted miR-155 targets that are increased in miR-155 KO macrophages, rassf6 is of particular interest since it is an inhibitor of the NFKB. ${ }^{30}$ MiR-155 has already been described to target $\mathrm{KB}-\mathrm{Ras} 1$, another NF-KB inhibitor, leading 
The role of miR-155 in cigarette smoke-induced pulmonary inflammation and...

E.G. De Smet et al.

430

Table 2. Differential gene expression between CS-exposed WT and miR-155 KO macrophages.

\begin{tabular}{|c|c|c|c|}
\hline & Full name & $\log \mathrm{FC}$ & adj.P.Val \\
\hline Slfn5 & Schlafen 5 & 4.353014048 & 0.043287057 \\
\hline Thy1 & Thymus cell antigen 1 & 4.072835002 & 0.014869036 \\
\hline C1qa & Complement component 1 , q subcomponent, alpha polypeptide & 3.850648794 & 0.03182286 \\
\hline Arg1 & Arginase 1 & 3.842642876 & 0.016280018 \\
\hline Gpc1 & Glypican 1 & 3.448654561 & 0.048050746 \\
\hline Tnfsf8 & Tumor necrosis factor (ligand) superfamily, member 8 & 3.447791401 & 0.012131543 \\
\hline Tcf7 & Transcription factor 7 & 3.401424707 & 0.037690132 \\
\hline Blnk & B cell linker & 3.382809764 & 0.039783275 \\
\hline Tmem176a & Transmembrane protein $176 \mathrm{~A}$ & 3.346217899 & 0.038885121 \\
\hline Stbd 1 & Starch binding domain 1 & 2.684533061 & 0.013985503 \\
\hline Stab1 & Stabilin 1 & 2.644164504 & 0.03073374 \\
\hline Adam19 & A disintegrin and metallopeptidase domain 19 & 2.493580603 & 0.047282169 \\
\hline Egr1 & Early growth response 1 & 2.315669725 & 0.013729936 \\
\hline Kenn4 & Potassium intermediate/small conductance calcium-activated channel, subfamily $\mathrm{N}$, member 4 & 2.31373034 & 0.03182286 \\
\hline $117 \mathrm{r}$ & Interleukin 7 receptor & 2.301429172 & 0.021883581 \\
\hline Stap1 & Signal transducing adaptor family member 1 & 2.17965702 & 0.017764318 \\
\hline Slamf7 & SLAM family member 7 & 1.955779445 & 0.018503067 \\
\hline Ccl17 & Chemokine ( $\mathrm{C}-\mathrm{C}$ motif) ligand 17 & 1.900853907 & 0.003096901 \\
\hline Nqo1 & $\mathrm{NAD}(\mathrm{P}) \mathrm{H}$ dehydrogenase, quinone 1 & 1.900618537 & 0.039415491 \\
\hline Mmp12 & Matrix metallopeptidase 12 & 1.785582158 & 0.003295582 \\
\hline Scd1 1 & Stearoyl-Coenzyme A desaturase 1 & 1.083412923 & $1.61 \mathrm{E}-06$ \\
\hline Idua & Iduronidase, alpha-L & 1.04583766 & $1.25 \mathrm{E}-05$ \\
\hline Cdkl2 & Cyclin-dependent kinase-like 2 & -1.080541203 & $8.28 \mathrm{E}-05$ \\
\hline Crim1 & Cysteine rich transmembrane BMP regulator 1 & -1.528354895 & 0.03182286 \\
\hline Sdk1 & Sidekick cell adhesion molecule 1 & -1.718722704 & 0.002973382 \\
\hline Gabbr1 & $\gamma$-aminobutyric acid B receptor 1 & -1.737620707 & 0.00317609 \\
\hline Bend4 & BEN domain containing 4 & -2.911261744 & 0.000857833 \\
\hline Rplp0.ps1 & Ribosomal protein, large, P0, pseudogene 1 & -3.686139093 & 0.033420139 \\
\hline Gm32098 & Predicted gene, 32098 & -3.769794542 & 0.03182286 \\
\hline Acacb & Acetyl-Coenzyme A carboxylase beta & -3.885530266 & 0.00317609 \\
\hline Gm14165 & Predicted gene, 14165 & -4.35232473 & 0.008003129 \\
\hline Gm9625 & Predicted gene, 9625 & -4.405631927 & $1.63 \mathrm{E}-09$ \\
\hline Myo18b & Myosin XVIIIb & -5.63251712 & 0.000725636 \\
\hline Slc10a6 & Solute carrier family 10 member 6 & -6.264293953 & 0.00032407 \\
\hline Rassf6 & Ras association (RalGDS/AF-6) domain family member 6 & -7.113025745 & 8.51E-07 \\
\hline Gm8730 & Predicted gene, 8730 & -8.73347261 & $2.62 \mathrm{E}-09$ \\
\hline
\end{tabular}

adj.P.Val adjusted $p$-value, $C S$ cigarette smoke, $F C$ fold change, KO knock-out, $W T$ wild type

Genes showing log2 fold-change differences $>1$ (in absolute value) and with Benjamini-Hochberg adjusted $p$-values smaller than 0.05 were considered statistically significant differentially expressed. Genes upregulated in CS-exposed WT macrophages compared to miR-155 KO macrophages have positive foldchange values. Genes upregulated in CS-exposed miR-155 KO macrophages compared to WT macrophages have negative fold-change values 
a

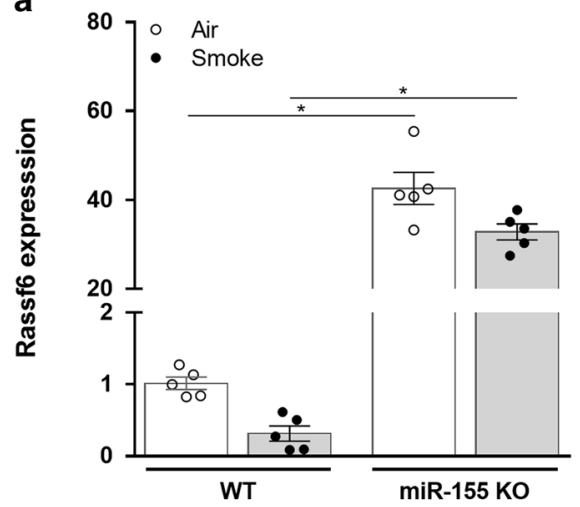

C

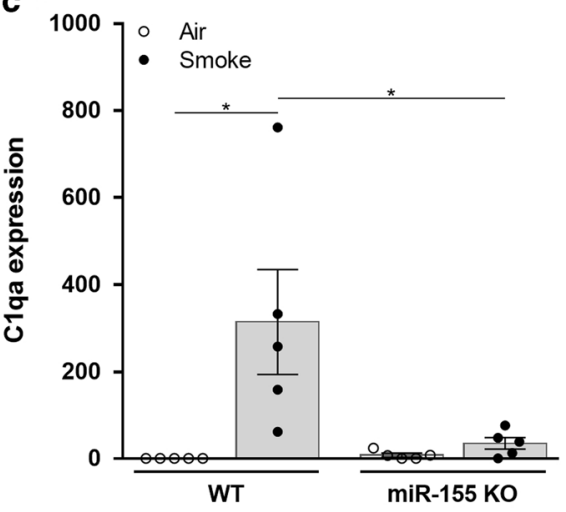

b

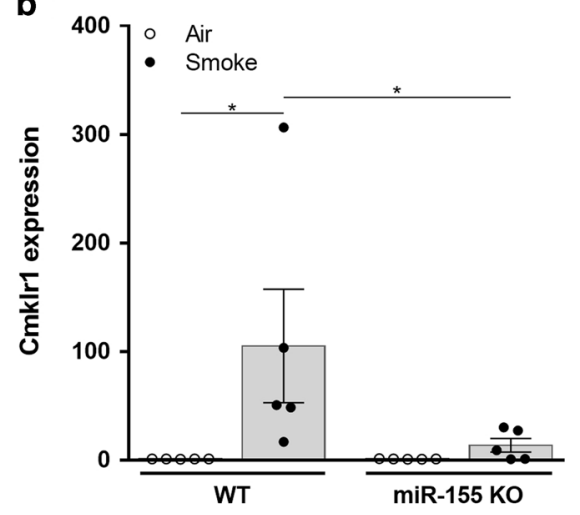

d

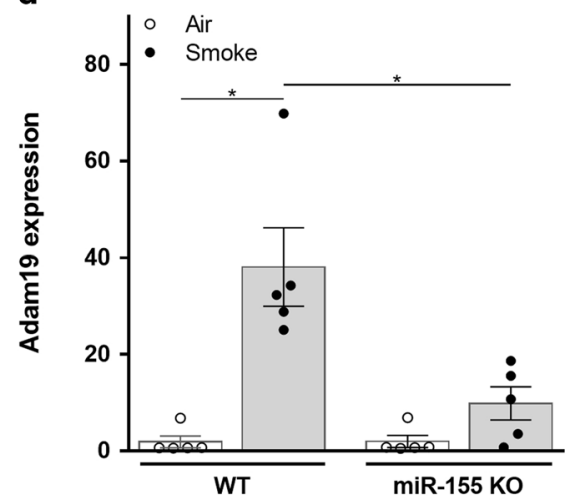

e

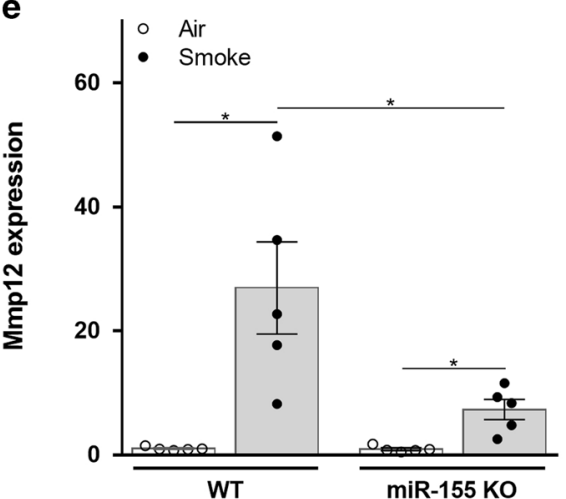

Fig. 5 Expression levels of a subset of differentially expressed genes in sorted alveolar macrophages from WT and miR-155 deficient mice. Expression levels of rassf6 (a), cmklr1 (b), c1qa (c), adam19 (d) and mmp12 (e) in alveolar macrophages from WT and miR-155 KO mice upon 4 weeks of air or cigarette smoke exposure, represented as fold change compared to air-exposed WT mice for which the mean expression is set as value 1 ( $n=5$ animals/group). Genes showing log2 fold-change differences greater than 1 (in absolute value) between the different pairwise comparisons and with Benjamini-Hochberg adjusted $p$-values $<0.05$ were considered differentially expressed.

to increased expression of G-CSF and TNF. ${ }^{46}$ However, by luciferase reporter assay we could not confirm rassf6 as a direct target of miR-155. Nevertheless, decreased NF-KB expression, due to increased expression of rassf6, in CS-exposed miR-155 KO mice could still contribute to the attenuated inflammatory profile in these mice. Additional studies are needed to elucidate the miR$155 /$ rassf6/NF-KB axis in the pathogenesis of COPD.

To overcome the effects of constitutive miR-155 KO mice, that lack miR-155 from birth, we instilled a miR-155 inhibitor in the lungs of WT mice during exposure to air or CS. This resulted in a significantly attenuated increase after CS-exposure of DCs and $\mathrm{CD}^{+}$and $\mathrm{CD}^{+}{ }^{+}$lymphocytes compared to mice receiving a scrambled control, suggesting a therapeutic potential of miR-155 inhibition in the pathogenesis of COPD.
Given that the expression of miR-155 is the highest in end-stage COPD and correlates significantly with lung function parameters of both airflow limitation and diffusing capacity, it is important to demonstrate the effect of miR-155 deficiency on lung function changes and development of emphysema. To this end, we intratracheally administered PPE to mice, mimicking the effects of elastases released by inflammatory cells during the chronic inflammation in COPD. Elastase-induced development of emphysema and increase in BAL neutrophils, DCs and lymphocytes was significantly attenuated in miR-155 KO mice. Moreover, in vivo lung function measurements revealed that the elastase-induced increase in compliance and reduction in elasticity as well as the left-upward shift in the pressure-volume-loops of elastase-treated mice (which is reminiscent of emphysema) were significantly 

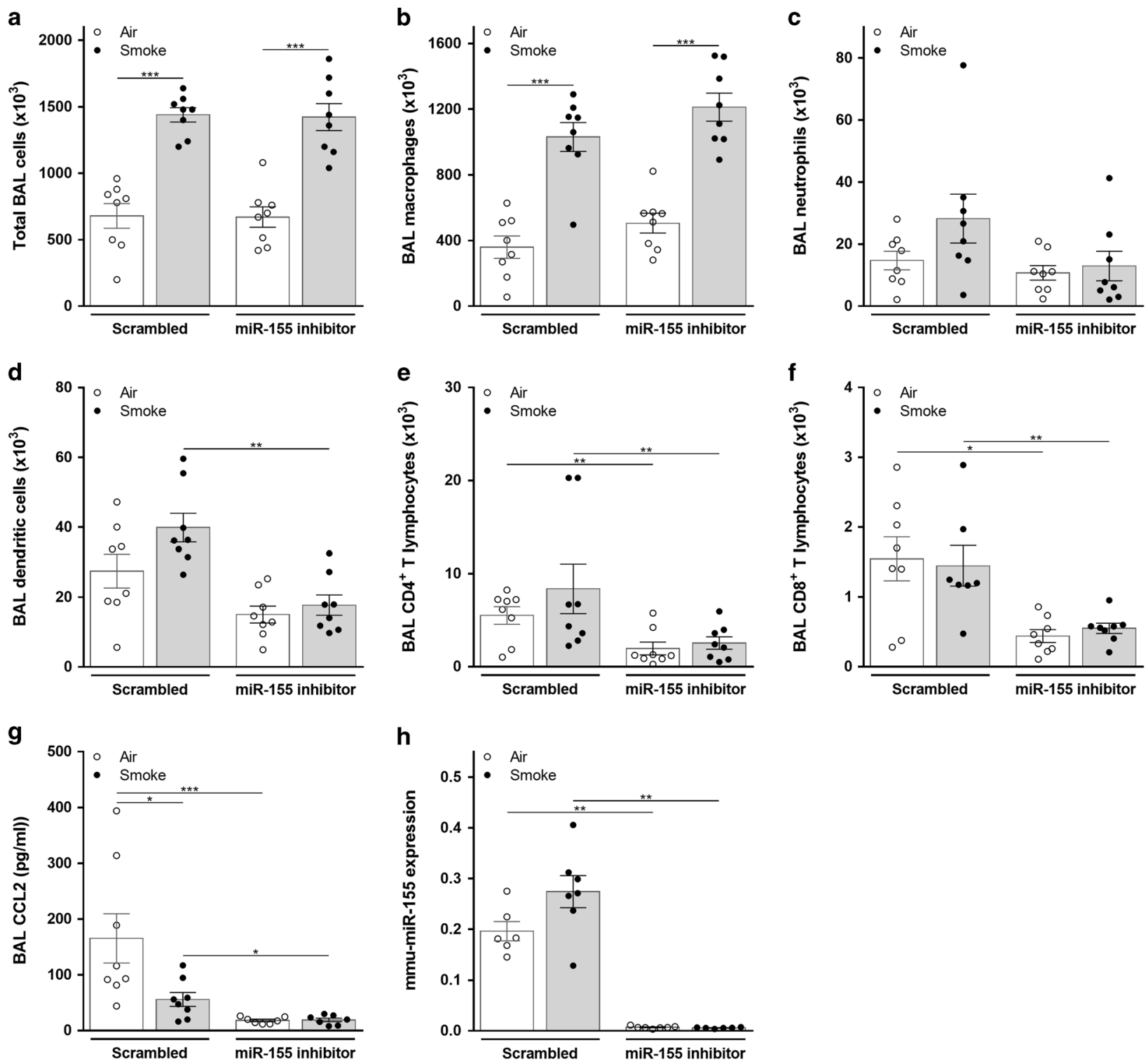

Fig. 6 Inflammatory cell numbers in the bronchoalveolar lavage fluid of air or cigarette smoke-exposed WT mice treated with a miR-155 inhibitor or scrambled control. Measurement by flow cytometry of total BAL cells (a), macrophages (b), neutrophils (c), dendritic cells (d), $\mathrm{CD}^{+}$T lymphocytes (e) and CD8 ${ }^{+}$T lymphocytes (f) and measurement by ELISA of CCL2 (g) in the bronchoalveolar lavage fluid of WT mice exposed to air or cigarette smoke for 4 weeks and intranasally instilled two times a week with a LNA-modified miR-155 inhibitor or scrambled control ( $n=7-8$ animals/group). Mmu-miR-155 levels measured by RT-qPCR in lung tissue of C57BL/6 mice exposed to air or cigarette smoke for 4 weeks and treated two times a week with an LNA-modified miR-155 inhibitor or scrambled control $(n=6-7$ animals/group) (h). Data are analyzed with the Kruskal-Wallis and Mann-Whitney $U$ test and expressed as mean \pm SEM $\left({ }^{*} P<0.05,{ }^{* *} P<0.01,{ }^{* *} P<0.001\right)$.

attenuated in miR-155 KO mice. Together, these results demonstrate that miR-155 deficiency not only impacts pulmonary inflammation but also histological and lung functional changes. However, studying the effect of miR-155 deficiency on histological changes induced by chronic exposure to CS remains an important research question.

In conclusion, we investigated the role of miR-155 in CSinduced inflammation and COPD. We demonstrated elevated expression of miR-155-5p in lungs of smokers and patients with COPD, as well as in lungs and alveolar macrophages of CSexposed mice. Next we showed that miR-155 plays a key role in CS-induced pulmonary inflammation, which is significantly attenuated in miR-155 KO mice and in WT mice treated with a specific miR-155 inhibitor. RNA sequencing in alveolar macrophages revealed differential expression of miR-155 targets according to exposure and genotype, highlighting an attenuation of the CS-induced increase in inflammation and proteases-related genes in miR-155 deficient mice. Finally, using the elastase model of emphysema, we demonstrate that miR-155 deficiency not only impacts pulmonary inflammation, but also histological and lung functional changes.

\section{METHODS}

Human lung tissue study population

Hsa-miR-155-5p expression was quantified by RT-qPCR in a study population of 92 subjects. In this study population 18 never smokers, 26 smokers without airflow limitation and 48 COPD patients were included (Table 1 ). Patients were considered as having COPD when their post-bronchodilator forced expiratory volume in $1 \mathrm{~s}$ [FEV ${ }_{1}$ ] on forced vital capacity [FVC] ratio was below 0.70 . When patients had quitted smoking for 

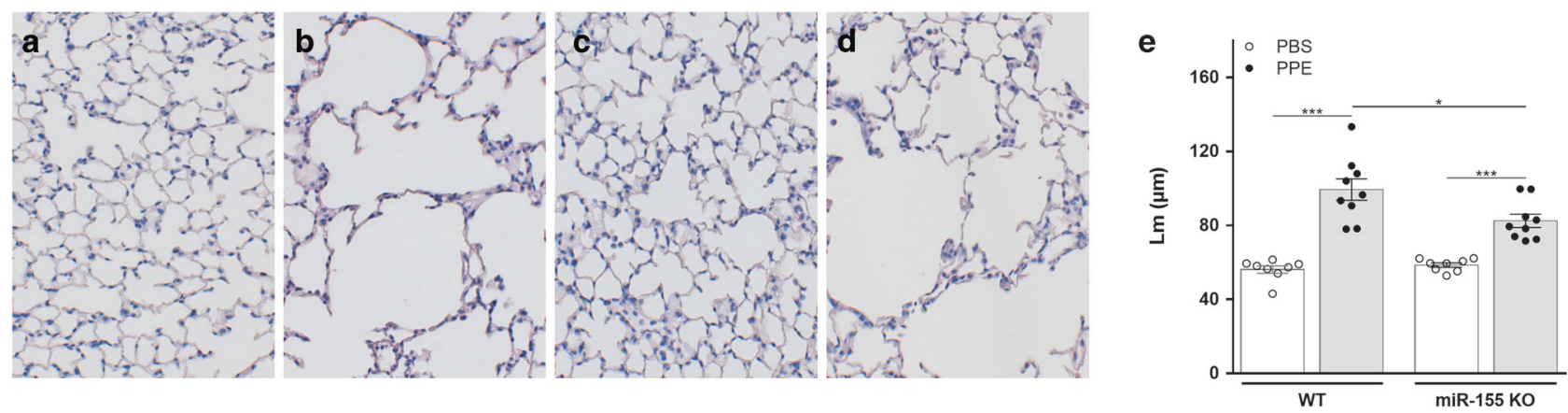

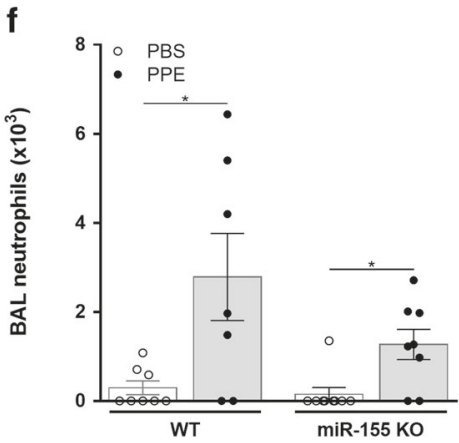

9

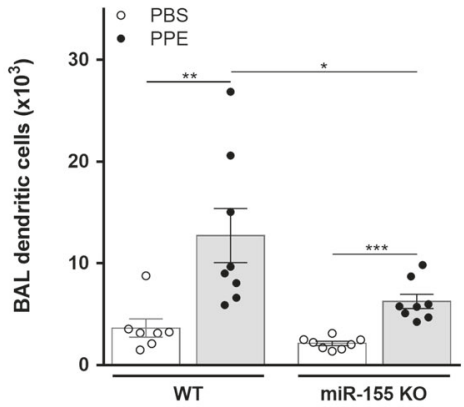

j

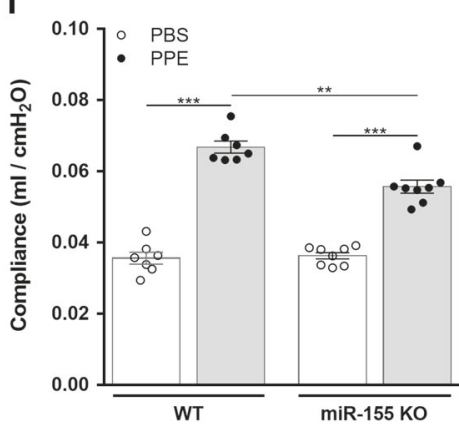

j

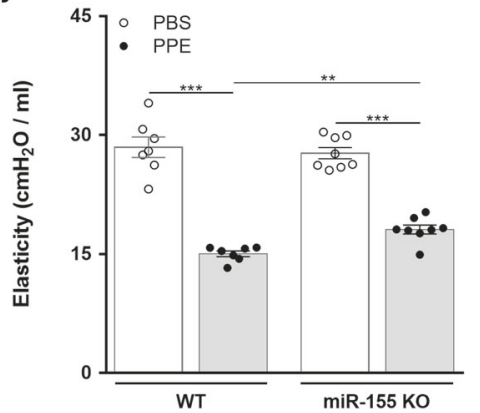

h

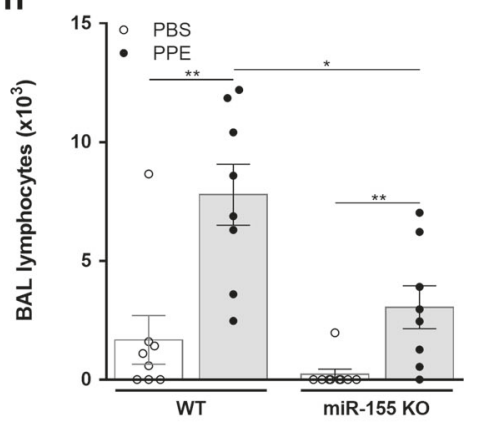

k

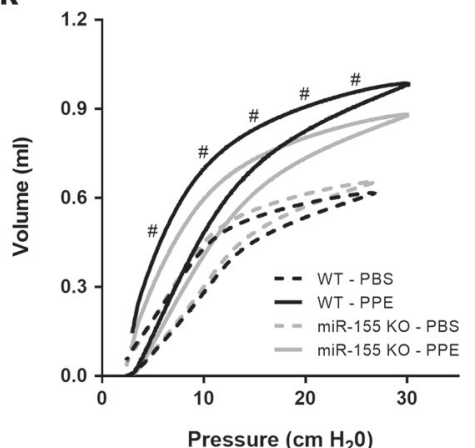

Fig. 7 Elastase-induced emphysema, inflammation and lung functional changes are attenuated in miR-155 deficient mice. Representative images of hematoxylin and eosin stained lung tissue of single PBS and porcine pancreatic elastase (PPE)-instilled C57BL/6 wild type (WT) and miR-155 knock-out (KO) mice (magnification x200). PBS-instilled WT mice (a), PPE-instilled WT mice (b), PBS-instilled miR-155 KO mice (c) and PPE-instilled miR-155 KO mice (d). Measurement of mean linear intercept (Lm) in lungs of single PBS and PPE-instilled wild type (WT) and miR$155 \mathrm{KO}$ mice (e). Measurement by flow cytometry of neutrophils (f), dendritic cells (g) and T-lymphocytes (h) in the bronchoalveolar lavage fluid of WT and miR-155 KO mice after a single PBS or PPE instillation (data are analyzed with the Kruskal-Wallis and Mann-Whitney $U$ test). Measurement by FlexiVent of compliance (i), elasticity (j) and pressure volume loops (k) of WT and miR-155 KO mice after three consecutive PBS or PPE instillations ("significantly different pressure-volume ratio at that given pressure, between WT and miR-155 KO PPE-instilled mice, as measured with a student $t$-test).

more than 1 year, they were defined as ex-smokers. Patients who experienced exacerbations within 2 months before the study were excluded, leaving only COPD patients with stable disease in the study population. In addition, patients receiving chemotherapy or radiotherapy in the last 6 months, patients with a diagnosis of mesothelioma, asthma or an infection of the upper or lower respiratory tract in the preceding 4 weeks were also excluded from the study. The lung resection specimens were obtained from 78 patients receiving surgery for solitary pulmonary tumors (Ghent University Hospital, Ghent, Belgium) and from 14 explant lungs of end-stage COPD patients undergoing lung transplantation (University Hospital Gasthuisberg, Leuven, Belgium). Lung tissue from the resection specimen at maximum distance from the tumor was collected by a pathologist and frozen at $-80^{\circ} \mathrm{C}$ in RNA later until further analysis. Written informed consent was obtained from all subjects. For this study, approval was received from the medical ethics committee of the Ghent University Hospital (2016/0132) and the University Hospital Gasthuisberg (S51577).

\section{Mice}

B6.Cg-Mir155 tm1.1Rsky J J (miR-155 KO) mice were obtained from the Jackson Laboratories (Bar Harbor, ME, USA) and bred at Charles River (L'Arbresle Cedex, France). C57BL/6 (wild type, WT) mice were obtained from Charles River. Male and female (for the lung function measurements) mice were used for the experiments. The mice were kept in a $12 \mathrm{~h}$ light-dark cycle and provided with a standard diet and chlorinated tap water ad libitum. The local ethics committee for animal experimentation of the faculty of Medicine and Health Sciences (Ghent University Hospital, Ghent, Belgium) approved all in vivo manipulations (ECD14/76 and ECD18/121). 
434

\section{CS exposure}

Mice were exposed to CS, as described previously. ${ }^{47-50}$ Briefly, mice ( $n=8-9$ mice/group) were exposed whole body to the tobacco smoke of five cigarettes (Reference Cigarette, 3R4F, University of Kentucky, Lexington, KY, USA) without filter and this four times a day with 30 min smoke-free intervals, 5 days a week for 4 weeks (subacute exposure) or 24 weeks (chronic exposure). Control mice were exposed to air.

In vivo perturbation of mmu-miR-155-5p in air and CS-exposed mice

Mmu-miR-155-5p was inhibited in lungs of mice exposed to air or CS for 4 weeks. $10 \mathrm{mg} / \mathrm{kg}$ of a locked nucleic acid (LNA) modified mmu-miR-155-5p inhibitor or a scrambled control (both from Exiqon, Vedbaek, Denmark) was intranasally instilled two times a week to mice exposed to air or CS for 4 weeks. The day after the last air or CS-exposure, BAL fluid was collected and analyzed.

Intratracheal instillation of porcine pancreatic elastase

Porcine pancreatic elastase (Sigma-Aldrich, Missouri, USA) was intratracheally administered with a dose of $0.5 \mathrm{U} / 25 \mathrm{~g}$ of body weight, dissolved in $50 \mu \mathrm{l}$ sterile PBS, following two protocols. In the first protocol, emphysema and inflammatory cells in BAL fluid were quantified 7 days after a single intratracheal instillation of elastase. In the second protocol mice received an instillation of elastase for 3 consecutive weeks and were subjected to in vivo lung function measurements (by FlexiVent) 7 days after the third and last instillation of elastase.

BAL and cell count

BAL fluid was obtained as previously described..$^{51}$ In summary, a tracheal cannula was placed and lungs were lavaged six times: three times using $300 \mu \mathrm{l}$ HBSS without $\mathrm{Ca}^{2+}$ and $\mathrm{Mg}^{2+}$, supplemented with $1 \%$ Bovine Serum Albumin and three times with $1 \mathrm{ml}$ HBSS without $\mathrm{Ca}^{2+}$ and $\mathrm{Mg}^{2+}$, supplemented with 0.6 mM EDTA. After the lavage fractions were pooled, total BAL cells were counted using a Bürker chamber and differential cell counts were obtained by May-Grünwald-Giemsa staining on cytocentrifuged preparations.

Lung function measurements

Using the Flexivent System (SCIREQ, Montreal, Canada), baseline lung function was examined invasively in tracheostomised anaesthetized mice. ${ }^{52}$ Pancuronium bromide (Inresa, Freiburg, Germany) was administered via the jugular vein to induce a neuromuscular blockade. The mice were ventilated with an average breathing frequency of 150 breaths/minute. After the mice were stable, dynamic compliance and elasticity were measured using a 'snapshot perturbation' manoeuvre and pressure-volume (PV) loops were generated.

Lung harvest and preparation of single-cell suspensions

After BAL was performed, the pulmonary and systemic circulation was rinsed with saline supplemented with $5 \mathrm{mM}$ EDTA. The small lobe of the right lung was used for RNA extraction. The middle lobe was used for preparation of lung homogenate (See Supplementary Information for more details). The major lobe was minced, digested and subjected to red blood lysis to obtain a single cell suspension. Cells were counted using a Z2 particle counter (Beckman-Coulter, Fullerton, CA, USA). The left lung was used for histology, as previously published. ${ }^{47}$

\section{Emphysema}

The mean linear intercept ( $\mathrm{Lm})$, a measurement of alveolar airspace enlargement, was quantified (as previously described ${ }^{47}$ ) to evaluate pulmonary emphysema. This quantification was performed on haematoxylin and eosin (H\&E) stained lung sections using image J software.
Flow cytometric analysis and cell sorting

Inflammatory cells in BAL fluid and lung single cell suspensions were quantified using flow cytometry (FACS LSR Fortessa, BD Biosciences, San Diego, CA, USA). For details regarding the staining and gating of the samples see Supplementary Information.

\section{RNA extraction}

RNA extraction of total lung and of FACS sorted alveolar macrophages was performed with the miRNeasy Mini Kit (Qiagen).

Quantification of miR-155-5p expression in lung tissue and FACS sorted alveolar macrophages and mRNA transcript levels in lung tissue

MiR-155-5p and mRNA expression levels were quantified by RTqPCR by use of a Lightcycler $96^{\circledR}$ SW 1.1 instrument (Roche, Basel, $\mathrm{CH})$. See Supplementary Information for more details.

Cytometric bead array/measurement of cytokines

The Mouse Enhanced Sensitivity Cytometric Bead Array (BD Biosciences) was used to measure protein levels of cytokines in BAL fluid and lung homogenate of mice. Different capture bead populations, each with distinct fluorescence intensities and coated with antibodies specific for IL-1 $\beta$, IL-6, IL-10, IL-17A, TNF and IFN- $\gamma$ were mixed and incubated with test samples or standards. Afterwards this mixture was incubated with PE-conjugated detection antibodies and acquisition of sample data was done using the FACS LSR Fortessa flow cytometer and analyzed using the FCAP Array Software (both BD Biosciences).

\section{ELISA}

CCL2, CXCL1 and CCL20 protein levels were determined in BAL fluid supernatant and lung homogenate of mice using commercially available ELISA kits (R\&D systems, Minneapolis, MN, USA) following the manufacturer's intructions.

\section{Sequencing of FACS sorted alveolar macrophages and data processing}

PolyA $^{+}$RNA sequencing libraries were prepared using the TruSeq mRNA library prep kit (Illumina, San Diego, CA, USA) according to the manufacturer's instructions. A total of $25 \mathrm{ng}$ of RNA was used as input for polyA selection, followed by fragmentation, reverse transcription and PCR amplification. Libraries were quantified and pooled for single-end sequencing on a NextSeq 500. For details regarding the analysis see Supplementary Information.

\section{Statistical analysis}

Statistical analysis was performed with Sigma Stat Software (SPSS version 25, Chicago, IL, USA). Following tests were performed: Kruskall-Wallis followed by post hoc Mann-Whitney U. P-values < 0.05 were considered significant. Normalization, log2transformation into counts per million (logCPM) and differential gene expression analyses of the sequencing data was performed using the $\mathrm{R}$ statistical programming language (version 3.5.1) and the limma package (version 3.38.2 ${ }^{53}$ ).

\section{ACKNOWLEDGEMENTS}

The authors would like to thank Greet Barbier, Indra De Borle, Katleen De Saedeleer, Anouck Goethals, Marie-Rose Mouton and Ann Neesen (Department of Respiratory Medicine, Ghent University) for their excellent technical assistance. We would also like to thank Prof. Wim Janssens and Dr. Bart Vanaudenaerde (Department of Pneumology, Catholic University of Leuven) for providing us with explant lungs of patients with severe COPD, and Prof. Dirk Elewaut (Department of Rheumatology, Ghent University) for the use of the FACSAria. The research described in this article was supported by the Concerted Research Action of the Ghent University (BOF/GOA, $01 \mathrm{G} 02714$ and 01G00819) and by the Fund for Scientific Research in Flanders (FWO Vlaanderen, G052518N and EOS-contract G0G2318N). F.M.V. and S.P. are postdoctoral researchers of the Fund for Scientific Research-Flanders and this work was 
The role of miR-155 in cigarette smoke-induced pulmonary inflammation and... E.G. De Smet et al.

further supported by a Special Research Fund (BOF) scholarship of Ghent University to Francisco Avila Cobos (BOF.DOC.2017.0026.01).

\section{AUTHOR CONTRIBUTIONS}

K.R.B., G.G.B., G.F.J., P.M., H.P.V.E., and E.G.D.S. conceived the project and designed the experiments; K.R.B., F.M.V., E.B., S.P., P.M., H.P.V.E., K.V., and E.G.D.S. conducted the experiments; F.A.C., K.R.B., G.G.B., G.F.J., F.M.V., T.M., H.P.V.E., and E.G.D.S. contributed to the data analysis and interpretation; E.G.D.S. wrote the manuscript and all authors contributed to writing and editing of the manuscript.

\section{ADDITIONAL INFORMATION}

The online version of this article (https://doi.org/10.1038/s41385-019-0241-6) contains supplementary material, which is available to authorized users.

Competing interests: G.F.J. reports grants and personal fees from AstraZeneca, grants from Chiesi, personal fees from Eureca, grants and personal fees from GlaxoSmithKline, grants and personal fees from Novartis, personal fees from Teva, outside the submitted work. T.M. reports grants from Belspo, grants from Ghent University, during the conduct of the study; personal fees from GlaxoSmithKline, outside the submitted work; and is shareholder of Oryzon Genomics. All other authors have nothing to disclose.

Publisher's note Springer Nature remains neutral with regard to jurisdictional claims in published maps and institutional affiliations.

\section{REFERENCES}

1. Vogelmeier, C. F. et al. Global Strategy for the Diagnosis, Management, and Prevention of Chronic Obstructive Lung Disease 2017 Report: GOLD Executive Summary. Eur. Respir. J. 2017; 49, pii:1700214.

2. WHO. Burden of disease. http://www.who.int/topics/global_burden_of_disease/ en/.

3. Murray, C. J. \& Lopez, A. D. Alternative projections of mortality and disability by cause 1990-2020: Global Burden of Disease Study. Lancet 349, 1498-1504 (1997).

4. Hogg, J. C. \& Timens, W. The pathology of chronic obstructive pulmonary disease. Annu. Rev. Pathol. 4, 435-459 (2009).

5. Abboud, R. T. \& Vimalanathan, S. Pathogenesis of COPD. Part I. The role of protease-antiprotease imbalance in emphysema. Int. J. Tuberculosis Lung Dis. 12, 361-367 (2008)

6. Yang, S. R. et al. Cigarette smoke induces proinflammatory cytokine release by activation of NF-kappaB and posttranslational modifications of histone deacetylase in macrophages. Am. J. Physiol. Lung Cell. Mol. Physiol. 291, L46-L57 (2006).

7. Vigorito, E., Kohlhaas, S., Lu, D. \& Leyland, R. miR-155: an ancient regulator of the immune system. Immunol. Rev. 253, 146-157 (2013).

8. Taganov, K. D., Boldin, M. P., Chang, K. J. \& Baltimore, D. NF-kappaB-dependent induction of microRNA miR-146, an inhibitor targeted to signaling proteins of innate immune responses. Proc. Natl Acad. Sci. USA 103, 12481-12486 (2006).

9. Thai, T. H. et al. Regulation of the germinal center response by microRNA-155. Science 316, 604-608 (2007).

10. O'Connell, R. M., Taganov, K. D., Boldin, M. P., Cheng, G. \& Baltimore, D. MicroRNA155 is induced during the macrophage inflammatory response. Proc. Natl Acad. Sci. USA 104, 1604-1609 (2007).

11. Haasch, D. et al. T cell activation induces a noncoding RNA transcript sensitive to inhibition by immunosuppressant drugs and encoded by the proto-oncogene, BIC. Cell Immunol. 217, 78-86 (2002).

12. Bluml, S. et al. Essential role of microRNA-155 in the pathogenesis of autoimmune arthritis in mice. Arthritis Rheum. 63, 1281-1288 (2011).

13. Singh, U. P. et al. miR-155 deficiency protects mice from experimental colitis by reducing $T$ helper type 1/type 17 responses. Immunology 143, 478-489 (2014).

14. Zhang, J. et al. MicroRNA-155 modulates Th1 and Th17 cell differentiation and is associated with multiple sclerosis and experimental autoimmune encephalomyelitis. J. Neuroimmunol. 266, 56-63 (2014).

15. O'Connell, R. M. et al. MicroRNA-155 promotes autoimmune inflammation by enhancing inflammatory T cell development. Immunity 33, 607-619 (2010).

16. Murugaiyan, G., Beynon, V., Mittal, A., Joller, N. \& Weiner, H. L. Silencing microRNA-155 ameliorates experimental autoimmune encephalomyelitis. J. Immunol. 187, 2213-2221 (2011).

17. Johansson, K., Malmhall, C., Ramos-Ramirez, P. \& Radinger, M. MicroRNA-155 is a critical regulator of type 2 innate lymphoid cells and IL-33 signaling in experimental models of allergic airway inflammation. J. Allergy Clin. Immunol. 139, 1007-1016, e9 (2016).
18. Malmhall, C. et al. MicroRNA-155 is essential for TH2-mediated allergen-induced eosinophilic inflammation in the lung. J. Allergy Clin. Immunol. 133, 1429-1438, e1427 (2014)

19. Zech, A. et al. MicroRNA-155 modulates P2R signaling and Th2 priming of dendritic cells during allergic airway inflammation in mice. Allergy 70, 1121-1129 (2015).

20. Wang, W. et al. Macrophage micro-RNA-155 promotes lipopolysaccharideinduced acute lung injury in mice and rats. Am. J. Physiol. Lung Cell Mol. Physiol. 311, L494-L506 (2016).

21. $\mathrm{Xu}, \mathrm{F}$. et al. Akt1-mediated regulation of macrophage polarization in a murine model of Staphylococcus aureus pulmonary infection. J. Infect. Dis. 208, 528-538 (2013).

22. Lu, Z. J. et al. MicroRNA-155 promotes the pathogenesis of experimental colitis by repressing SHIP-1 expression. World J. Gastroenterol. 23, 976-985 (2017).

23. Barnes, P. J. Alveolar macrophages as orchestrators of COPD. COPD 1, 59-70 (2004).

24. Culpitt, S. V. et al. Impaired inhibition by dexamethasone of cytokine release by alveolar macrophages from patients with chronic obstructive pulmonary disease. Am. J. Respir. Crit. Care Med 167, 24-31 (2003).

25. Finlay, G. A. et al. Matrix metalloproteinase expression and production by alveolar macrophages in emphysema. Am. J. respiratory Crit. Care Med. 156, 240-247 (1997).

26. Shapiro, S. D. The macrophage in chronic obstructive pulmonary disease. Am. J. Respir. Crit. Care Med. 160(5 Pt 2), S29-S32 (1999).

27. Conickx, G. et al. MicroRNA Profiling Reveals a Role for MicroRNA-218-5p in the Pathogenesis of Chronic Obstructive Pulmonary Disease. Am. J. Respir. Crit. Care Med. 195, 43-56 (2017).

28. Conickx, G. et al. microRNA profiling in lung tissue and bronchoalveolar lavage of cigarette smoke-exposed mice and in COPD patients: a translational approach. Sci. Rep. 7, 12871 (2017).

29. The miRNA body-map. http://mellfire.ugent.be/public/body_map/index.php.

30. Allen, N. P. et al. RASSF6 is a novel member of the RASSF family of tumor suppressors. Oncogene 26, 6203-6211 (2007).

31. Elmesmari, A. et al. MicroRNA-155 regulates monocyte chemokine and chemokine receptor expression in Rheumatoid Arthritis. Rheumatology 55, 2056-2065 (2016).

32. Ma, L., Xue, H. B., Wang, F., Shu, C. M. \& Zhang, J. H. MicroRNA-155 may be involved in the pathogenesis of atopic dermatitis by modulating the differentiation and function of T helper type 17 (Th17) cells. Clin. Exp. Immunol. 181, 142-149 (2015).

33. Kurowska-Stolarska, M. et al. The role of microRNA-155/liver X receptor pathway in experimental and idiopathic pulmonary fibrosis. J. Allergy Clin. Immunol. 139, 1946-1956 (2017).

34. Bruning, U. et al. MicroRNA-155 promotes resolution of hypoxia-inducible factor 1alpha activity during prolonged hypoxia. Mol. Cell Biol. 31, 4087-4096 (2011).

35. Nazari-Jahantigh, M. et al. MicroRNA-155 promotes atherosclerosis by repressing Bcl6 in macrophages. J. Clin. Invest 122, 4190-4202 (2012).

36. O'Connell, R. M., Chaudhuri, A. A., Rao, D. S. \& Baltimore, D. Inositol phosphatase SHIP1 is a primary target of miR-155. Proc. Natl Acad. Sci. USA 106, 7113-7118 (2009).

37. Androulidaki, A. et al. The kinase Akt1 controls macrophage response to lipopolysaccharide by regulating microRNAs. Immunity 31, 220-231 (2009).

38. Bandyopadhyay, S., Long, M. E. \& Allen, L. A. Differential expression of microRNAs in Francisella tularensis-infected human macrophages: miR-155-dependent downregulation of MyD88 inhibits the inflammatory response. PLOS ONE 9, e109525 (2014).

39. Li, Y., Ma, D., Wang, Z. \& Yang, J. MicroRNA-155 deficiency in Kupffer cells Ameliorates liver ischemia-reperfusion injury in mice. Transplantation 101, 1600-1608 (2017).

40. Mann, M. et al. An NF-kappaB-microRNA regulatory network tunes macrophage inflammatory responses. Nat. Commun. 8, 851 (2017).

41. Bohlson, S. S., Fraser, D. A. \& Tenner, A. J. Complement proteins C1q and MBL are pattern recognition molecules that signal immediate and long-term protective immune functions. Mol. Immunol. 44, 33-43 (2007).

42. Demoor, T. et al. The role of ChemR23 in the induction and resolution of cigarette smoke-induced inflammation. J. Immunol. 186, 5457-5467 (2011).

43. Hautamaki, R. D., Kobayashi, D. K., Senior, R. M. \& Shapiro, S. D. Requirement for macrophage elastase for cigarette smoke-induced emphysema in mice. Science 277, 2002-2004 (1997).

44. Churg, A. et al. Macrophage metalloelastase mediates acute cigarette smokeinduced inflammation via tumor necrosis factor-alpha release. Am. J. Respiratory Crit. Care Med. 167, 1083-1089 (2003).

45. Hancock, D. B. et al. Meta-analyses of genome-wide association studies identify multiple loci associated with pulmonary function. Nat. Genet. 42, 45-52 (2010). 
The role of miR-155 in cigarette smoke-induced pulmonary inflammation and...

E.G. De Smet et al.

436

46. Wang, L. et al. Notch-dependent repression of miR-155 in the bone marrow niche regulates hematopoiesis in an NF-kappaB-dependent manner. Cell Stem Cell 15, 51-65 (2014).

47. D'Hulst, A. I., Vermaelen, K. Y., Brusselle, G. G., Joos, G. F. \& Pauwels, R. A. Time course of cigarette smoke-induced pulmonary inflammation in mice. Eur. Respir. J. 26, 204-213 (2005).

48. Seys, L. J. et al. Role of B cell-activating factor in chronic obstructive pulmonary disease. Am. J. Respir. Crit. Care Med. 192, 706-718 (2015).

49. Bracke, K. R. et al. Role of CXCL13 in cigarette smoke-induced lymphoid follicle formation and chronic obstructive pulmonary disease. Am. J. Respir. Crit. Care Med. 188, 343-355 (2013).
50. Bracke, K. R. et al. Cigarette smoke-induced pulmonary inflammation and emphysema are attenuated in CCR6-deficient mice. J. Immunol. 177, 4350-4359 (2006).

51. Bracke, K. R. et al. Cigarette smoke-induced pulmonary inflammation, but not airway remodelling, is attenuated in chemokine receptor 5-deficient mice. Clin. Exp. Allergy 37, 1467-1479 (2007).

52. Lanckacker, E. A. et al. Short cigarette smoke exposure facilitates sensitisation and asthma development in mice. Eur. Respir. J. 41, 1189-1199 (2013).

53. Ritchie, M. E. et al. limma powers differential expression analyses for RNA-sequencing and microarray studies. Nucleic Acids Res. 43, e47 (2015). 